\title{
PS-meson form factors in relativistic quantum mechanics and constraints from covariant space-time translations
}

\author{
Bertrand DESPLANQUES* \\ CNRS/IN2P3, INPG, France \\ E-mail: desplanq@lpsc.in2p3.fr \\ Yu-bing DONG \\ Institute of High Energy Physics, Chinese Academy of Science, \\ Beijing 100049, P. R. China \\ Theoretical Physics Center for Science Facilities (TPCSF), CAS, \\ Beijing 100049, P. R. China \\ E-mail: dongyb@mail.ihep.ac.cn
}

Laboratoire de Physique Subatomique et Cosmologie, Université Joseph Fourier Grenoble 1,

\begin{abstract}
The role of Poincaré covariant space-time translations is investigated in the case of the pseudoscalar-meson charge form factors calculated within a relativistic quantum mechanics framework. It is shown that this role extends beyond the standard energy-momentum conservation, which is accounted for in all works based on this general approach. It implies constraints that have been largely ignored until now but should be nevertheless fulfilled to ensure the full Poincaré covariance. The violation of these constraints, which is more or less important depending on the form of relativistic quantum mechanics that is employed, points to the validity of using a single-particle current, which is generally assumed in calculations of form factors. In short, these constraints concern the relation of the momentum transferred to the constituents to the one transferred to the system, which most often differ in relativistic quantum mechanics while they are equal in field theory. How to account for the constraints, as well as restoring the equivalence of different relativistic quantum mechanics approaches in estimating form factors, is discussed. It is mentioned that the result so obtained can be identified to a dispersion-relation one. A short conclusion relative to the underlying dynamics is given in the pion case.
\end{abstract}

Light Cone 2010: Relativistic Hadronic and Particle Physics

June 14-18, 2010

Valencia, Spain

*Speaker. 


\section{Introduction}

Calculations of form factors in relativistic quantum mechanics (RQM) generally imply the choice of a particular hypersurface to describe the process under consideration. Among these hypersurfaces, those that exhibit some symmetry properties have retained the attention and have given rise to various forms of relativity, first considered by Dirac [1]. The relativistic character of the approach supposes that the Poincaré algebra, implying rotation, boost and space-time translation operators, be satisfied. The construction of this algebra within the RQM framework was first performed by Bakamjian and Thomas for the instant form [2] and extended later on to other forms [3]. It relies on a mass operator that has to fulfill general conditions but does not need to be specified otherwise. This mass operator can be used in any form and can involve further relativistic effects that are not required for ensuring Poincaré covariance properties relevant for describing some state. Calculations of form factors involve states with different momenta. Poincaré covariance for such a process implies that form factors should not depend on the hypersurface chosen to describe the dynamics [4], which is a matter of convenience. This equivalence of different approaches then supposes, generally, to account for the contribution of many-particle currents at all orders in the interaction [4]. In practice however, calculations are based on a single-particle current and, as a result, form factors may depend on the approach. Restoring the equivalence of different approaches is a necessary task prior to any comparison of estimates to measurements. In the absence of many-particle currents, one could tentatively discriminate between the approaches by checking whether they fulfill Poincare covariance properties. Most often, the transformations of form factors under rotations or boosts are discussed. The role of space-time translations is limited to the total energy-momentum conservation, which is assumed in all cases. In this contribution, we show that this conservation property does not exhaust all covariance properties of currents under space-time translations (sect. 2). The further constraints that they imply [5] are considered in detail (sect. 3). We describe a way to account for them and discuss their role in restoring the equivalence of different RQM approaches, what is made here for pseudoscalar mesons (sect. 4).

Due to a lack of space, we mainly concentrate in the present contribution on the essential points underlying our approach for restoring the equivalence of different implementations of relativistic quantum mechanics in calculating form factors. We refer to refs. $[6,7,8]$ for technical details.

\section{Constraints from transformations of currents under space-time translations}

Covariant transformations of currents under space-time translations imply the relation:

$$
J^{v}(x)(\text { or } S(x))=e^{i P \cdot x}\left(J^{v}(0)(\text { or } S(0))\right) e^{-i P \cdot x} .
$$

When it is taken between eigenstates of the total momentum operator, $P^{\mu}$, it allows one to factorize the dependence on the space-time coordinate, $x$, at the r.h.s.:

$$
<i \mid J^{v}(x)(\text { or } S(x))\left|f>=e^{i\left(P_{i}-P_{f}\right) \cdot x}<i\right| J^{v}(0)(\text { or } S(0)) \mid f>.
$$

Combined with the $e^{i q \cdot x}$ function representing an external field carrying the momentum $q^{\mu}$, one gets the current momentum-energy conservation relation:

$$
\left(P_{f}-P_{i}\right)^{\mu}=q^{\mu},
$$


either under the assumption of space-time translation invariance or by performing an integration over $x$. At this point, we observe that the above relation does not imply any close relation for $q^{\mu}$ and the momentum transferred to the constituents, $\left(p_{f}-p_{i}\right)^{\mu}$, in contrast to field theory where an equality is expected. We also observe that relation (2.2) tells nothing on whether the current at $x=0$ can be restricted to a single-particle one though this is assumed in most works independently of the RQM approach.

The question arises of whether relations stemming from the most general transformations of currents under space-time translations, eq. (2.1), implying in particular the vicinity of the point $x=0$, can shed some light on the above observations. Among the numerous possible relations [5], the following one is especially relevant:

$$
\left[P_{\mu},\left[P^{\mu}, J^{v}(x)\right]\right]=-\partial_{\mu} \partial^{\mu} J^{v}(x),\left[P_{\mu},\left[P^{\mu}, S(x)\right]\right]=-\partial_{\mu} \partial^{\mu} S(x) .
$$

Considering the matrix element of these relations between eigenstates of $P^{\mu}$ and assuming a singleparticle current, one should satisfy the relation:

$$
<\mid q^{2} J^{v}(0)(\text { or } S(0))|>=<|\left(p_{i}-p_{f}\right)^{2} J^{v}(0)(\text { or } S(0)) \mid>.
$$

It is easily seen that this equation cannot be generally fulfilled in RQM approaches as, most often, $q^{2} \neq\left(p_{i}-p_{f}\right)^{2}$ (see fig. 1 for a graphical representation). This implies that the assumption of a single-particle current is inconsistent with the covariance properties from space-time translations. The current, $J^{v}(0)$ (or $S(0)$ ), besides a single-particle component, should therefore also contain many-particle components which, until now, have been ignored. There is however one exception. In the standard front-form case $\left(q^{+}=q \cdot \omega=0\right)$, it turns out that the relation $q^{2}=\left(p_{i}-p_{f}\right)^{2}$ is fulfilled, implying that eq. (2.5) is always satisfied, while being limited to a single-particle current.

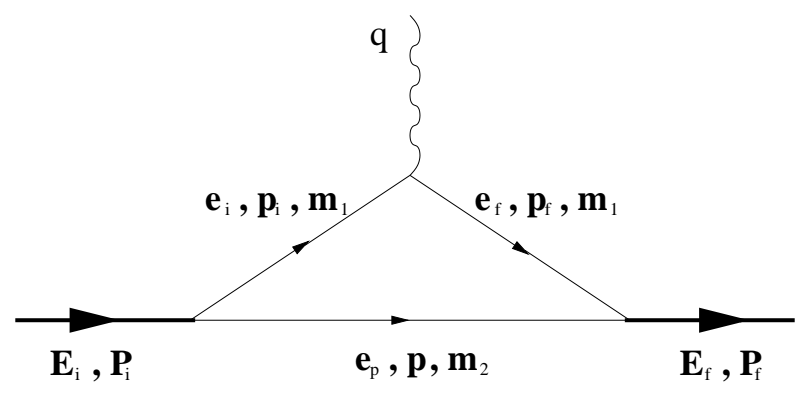

Figure 1: Representation of the interaction with an external probe and kinematical definitions

\section{Implementation of the constraints}

It is generally expected that many-particle currents at all orders in the interaction are required to fulfill the full Poincare covariance properties, possibly allowing one to restore the equivalence of different approaches for calculating form factors. As, for numerical reasons, the simplest twoparticle currents are rarely considered $[9,10]$, the above task seems to be out of reach a fortiori. However, the many-particle currents of interest here represent a specific subset, with a well defined role, namely accounting for constraints from covariant space-time translations. Moreover, with 
this respect, the front-form approach is consistent with a single-particle current. One can therefore infer that there may be some trick allowing one to sum up the contributions of the many-particle currents so that the single-particle current structure appropriate for the front-form approach be recovered. The trick we found is suggested by examining expressions of form factors. They show that the factors multiplying $Q$ in different approaches, 1 and $\frac{2 e_{k}}{M}$ in the most striking cases, differ by terms that have typically an interaction character [10] and are a signature of describing physics on different underlying hypersurfaces. The idea is to multiply $\mathrm{Q}$ by a factor $\alpha$ so that to account for the further interaction currents expected to restore the equivalence of different approaches. The factor $\alpha$ is determined by requiring that the squared momentum transferred to the system, $q^{2}$, be equal to the one for the constituents, denoted " $\left(p_{i}-p_{f}\right)$ "' . The equation to be solved is typically given by:

$$
\begin{aligned}
q^{2} & ="\left[\left(P_{i}-P_{f}\right)^{2}+2\left(\Delta_{i}-\Delta_{f}\right)\left(P_{i}-P_{f}\right) \cdot \xi+\left(\Delta_{i}-\Delta_{f}\right)^{2} \xi^{2}\right] " \\
& =\alpha^{2} q^{2}-2 \alpha "\left(\Delta_{i}-\Delta_{f}\right) " q \cdot \xi+"\left(\Delta_{i}-\Delta_{f}\right)^{2} " \xi^{2},
\end{aligned}
$$

where $\Delta$, which represents an interaction effect, also depends on $\alpha$. Explicit expressions of $\alpha$ can be found for different forms. Expressions for form factors, taking into account the effect of constraints motivated by space-time translation properties, can then be obtained [6,7]. The manyparticle character of corrections to the current at all orders of the interaction could be checked by expanding these expressions in terms of $\Delta$. It is worthwhile to notice that, for the standard frontform where $\xi^{2}=0, q \cdot \xi$ (or $\left.q^{+}\right)=0$, the factor $\alpha$ is equal to 1 and, therefore, results for the form factors are unchanged. Moreover, the expression of the single particle-current in other forms, before accounting for the constraints, may slightly differ from what is expected in an impulse approximation but the difference involves effects that are typically interaction ones. Its choice ensures that results for form factors are Lorentz invariant.

\section{Restoration of the equivalence: numerical illustration and comments}
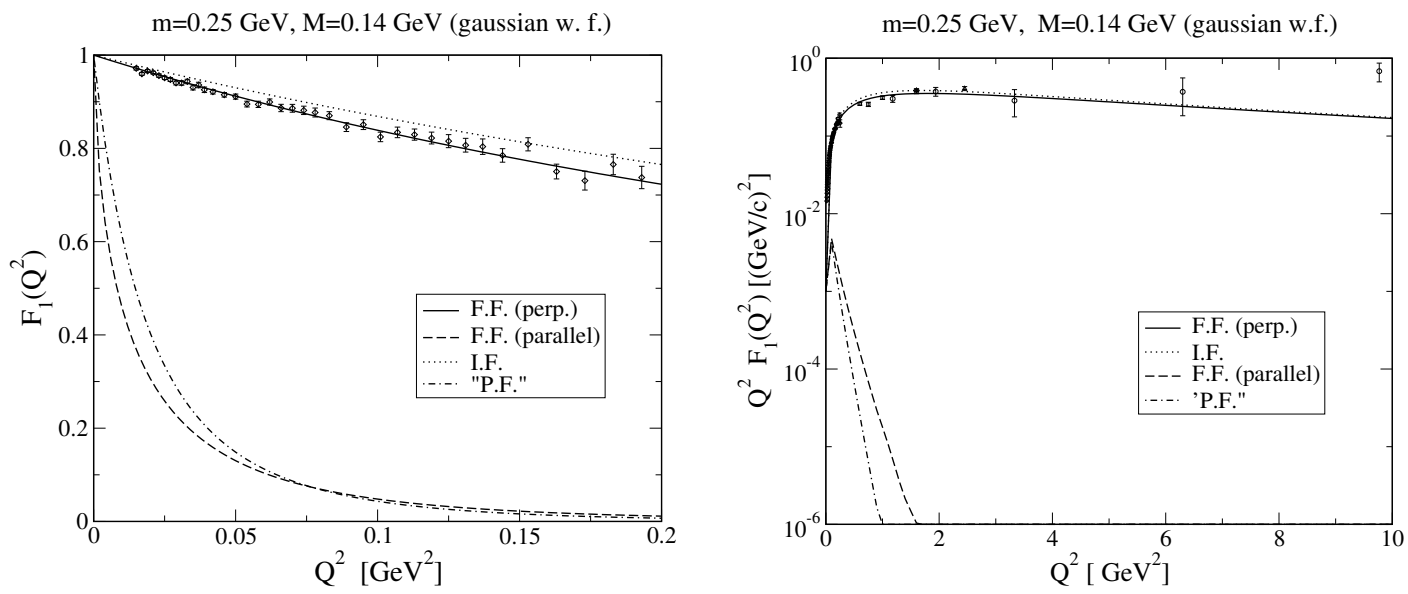

Figure 2: Pion charge form factor at low and intermediate $Q^{2}$, without and with the effect of constraints considered in this work. In the last case, all curves coincide with the standard front-form one (F.F. (perp.)). 
To illustrate effects of the restoration of properties related to space-time translations, we calculated the charge form factor for both the pion and kaon mesons using a Gaussian wave function which approximately accounts for the confinement interaction (string tension equal to $0.2 \mathrm{GeV}^{2}$ ). The quark mass is fixed by fitting the meson decay constant. We present in fig. 2 results for the pion, at low $Q^{2}$ in the left panel and at intermediate $Q^{2}$ in the right panel. The left panel shows the sensitivity to the charge radius. In the right panel, the form factor has been multiplied by $Q^{2}$ as often done on the basis that the corresponding product could tend to a constant asymptotically (up to $\log$ terms). We nevertheless stress that the two-particle contribution to the current which could reproduce this behavior $[6,9]$ is not included in the results presented here. The various curves shown in the figure correspond to different forms or different momentum configurations and are obtained for the Breit frame (F.F. (perp.): standard front form with $q^{+}=0$; F.F. (parallel): front form with $\vec{q}$ parallel to the front orientation; I.F.: standard instant form [2]; "P.F.": "instant form with the symmetry properties of the Dirac point form" [11]). We do not include results for the Dirac point form (hyperboloid hypersurface), which fall between the two front-form curves [12].

Examination of results without the effect of constraints considered in this work evidences striking features. While the standard front-form and instant-form results are close to each other, they show tremendous discrepancies with the other two curves. The fast fall off of form factors at low $Q^{2}$ in the last cases reflects a somewhat paradoxical $1 / M^{2}$ dependence of the pion squared charge radius. Though there was no intent to make a detailed comparison to measurements, it is clear that the two first approaches do relatively well, despite the corresponding results are not Lorentz invariant a priori while the "P.F." one, which evidences this property, does very badly. After incorporating the effect of constraints, all curves are found to coincide with the standard front-form one, which is not changed. The paradox of a charge radius tending to infinity while the mass of the system goes to zero (or the interaction is increased) has disappeared, suggesting that the effect of constraints we considered corrects for the breaking of some symmetry related to spacetime translations. For the sake of the illustration, we presented results obtained in the Breit frame but we could choose any frame as well. We stress that the results accounting for the constraints are independent of both the frame and the orientation of the hyperplane $\xi^{\mu}$. These properties can be readily checked by looking at the expression that the pion charge form factor takes after making a change of variables and integrating over one of them:

$$
F_{1}\left(Q^{2}\right)=\frac{1}{N} \int d \bar{s} d\left(\frac{s_{i}-s_{f}}{Q}\right) \phi\left(s_{i}\right) \phi\left(s_{f}\right) \frac{2 \sqrt{s_{i} s_{f}} \theta\left(\frac{s_{i} s_{f}}{D}-m^{2}\right)}{D \sqrt{D}} .
$$

This expression agrees with a dispersion-relation one found prior to this work by Melikhov [13] but disagrees by a factor $\left(s_{i}+s_{f}+Q^{2}\right) /\left(2 \sqrt{s_{i} s_{f}}\right)$ with the one obtained by Krutov and Troitsky [14]. The discrepancy factor in this case is the same as for scalar constituents [8].

We would like to mention that getting for all approaches the same expression of the form factor in terms of the Mandelstam variables $s_{i}, s_{f}$, eq. (4.1), does not imply that the expression in terms of the spectator momentum, $\vec{p}$, is the same. This is illustrated in fig. 3 where we show the integrands in terms of the quantity $p=|\vec{p}|$ corresponding to results presented in fig. 2 at two momentum transfers $Q^{2}=1$ and $Q^{2}=10 \mathrm{GeV}^{2}$. The change of variables that allows one to make the integration over one of them and reduce a 3-dimensional integration to a 2-dimensional one is specific of each approach and is non-trivial. It allows one to concentrate all the explicit dependence 

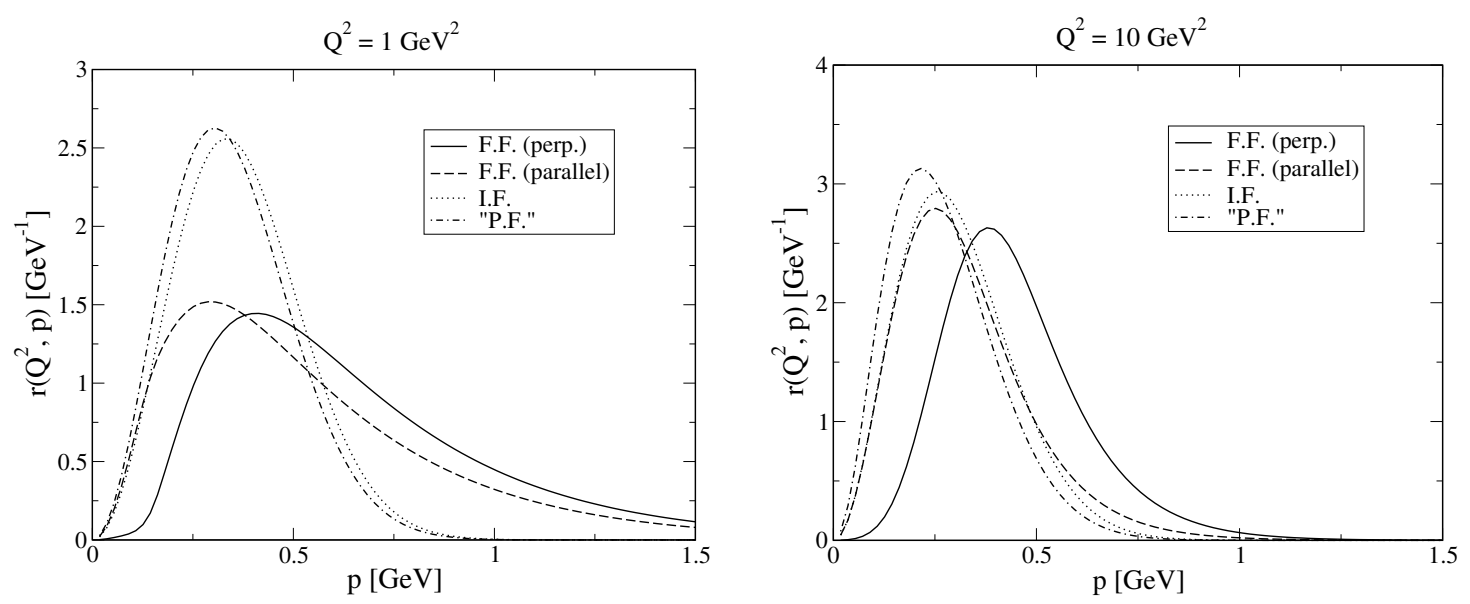

Figure 3: Representation of the integrand in different approaches as a function of the spectator momentum, $p=|\vec{p}|$ (see ref. [6] for a precise definition of the quantities shown in the figure).

of the integrand on the frame or on the front orientation in a new variable that can be integrated over, leaving only $s_{i}$ and $s_{f}$ as integration variables.

The pseudoscalar mesons, besides a charge form factor, have a Lorentz scalar form factor. Results in this case are qualitatively very similar to the charge form factor ones. Charge form factors were calculated taking into account that the constituents have the spin structure appropriate to quarks (spin-1/2). They of course differ from those for scalar constituents [8] but, roughly, we observe the same qualitative features (the largest effects involve the wave function). Charge form factors have also been calculated for the kaon meson [6]. The effect of constraints is not as large as for the pion case. The reason is to be looked for in the kaon mass. It is reminded that the effect, in the most striking cases, involves terms $2 e_{k} / M$, which is smaller for the kaon than for the pion.

\section{Conclusion}

We considered in this work properties related to Poincare covariant space-time translations in RQM approaches for the calculation of form factors of pseudoscalar mesons. Apart from the standard front form with $q^{+}=0$, all other RQM approaches need to be completed for the manyparticle currents that the above properties, generally ignored, imply. When this is done, it is found that discrepancies between different RQM approaches for calculating form factors of pseudoscalar mesons can be removed, showing that the role of space-time translations extends beyond the standard energy-momentum conservation. It is also found that these results could coincide with those of a dispersion-relation approach. Results are qualitatively very similar to those obtained in a previous work with scalar constituents [8]. Altogether, all aspects of the Poincaré group (rotations, boosts but also space-time translations) are essential in getting reliable estimates of form factors as far as the implementation of relativity is concerned. While this important result is obtained by considering many-particle currents, we would like to emphasize that these ones represent a minimal subset that allows one to fulfill expected transformation properties of observables when the generators of the Poincare group are applied to them. It is also noticed that the invariance of form factors under Lorentz transformations alone is by far not sufficient to guarantee the validity of an 
approach, as evidenced by the "P.F." example. This observation raises another question. The fast fall off of the pion form factor in this last approach is obtained in a truncated front-form fieldtheory calculation $[15,16,17]$, where the effect is interpretated as due to missing the contribution of zero modes related to the breaking of the rotational symmetry, as well as in an instant form with an infinite-momentum configuration, where, instead, the rotational symmetry is fulfilled. Results presented in this work would rather suggest that the fast fall off of the pion charge form factor in some calculations is due to the fact that, besides breaking rotational or boost invariance, they simultaneously miss part of transformation properties under space-time translations. This aspect should deserve specific studies. We mentioned that restoring the equivalence of different approaches was requiring the contribution of a subset of many-particle currents. As an example of further currents, we could mention those allowing one to reproduce the expected asymptotic behavior of the pion charge form factor, which was recently determined in a RQM framework [9].

Having determined a set of results that are independent of the chosen implementation of relativity, one can proceed to their comparison with measurements. This comparison can provide information on the mass operator that can be taken to be the same in all approaches. This operator possibly involves relativistic effects that differ from those ensuring the equivalence of approaches for the calculation of form factors. Taking the standard front-form results as representative of these common results, we find that the calculated charge form factor does rather well in the pion case. Results for the kaon charge form factor are not so good but, due to various uncertainties, it is difficult to claim that there is a real discrepancy. In any case however, there are many reasons to consider the above results for the charge form factors as suspicious, especially in the pion case where there is a relatively good agreement. The calculation misses the whole physics related to the one-gluon exchange in both the interaction entering the mass operator and in the currents. Their contributions were considered in various works but, whatever the approach, accounting for them tends to overshoot measurements in the intermediate $Q^{2}$ range. Part of the solution to this problem probably requires to take into account in the determination of the mass operator corrections that correspond to retardation effects in a field-theory approach. These effects decrease the strength of the one-gluon exchange interaction obtained in the instantaneous approximation. Similar effects were found to be relevant for a system made of scalar constituents [8]. Another part of the solution to the overshooting problem may require the introduction of some quark form factors $[18,19,20$, 21]. Its choice should however take into account that part of the effect underlying the vector meson dominance phenomenology is already included. Moreover, its effect should be consistent with the expectation of a vanishing in the limit of a zero value for the QCD coupling, $\alpha_{s}$. With this respect, the approach developed in ref. [20] could be more appropriate.

The present work was extending to spin-1/2 constituents a previous one for scalar constituents. Further work along present lines would involve inelastic and time-like processes as well as nonzero spin systems such as meson resonances or the nucleon and its resonances. This task could require more elaboration. Let's mention that in the nucleon case, large effects from the constraints considered in this work are expected too though not as large as for the pion. The factor that determines the size of these effects is roughly a half of what it is in the pion case, where the corresponding factor, $2 e_{k} / M$, can be as large as 6 at low $Q^{2}$. Smaller effects are expected for resonances however. 


\section{Acknowledgments}

This work is partly supported by the National Sciences Foundations of China under grants No. 10775148,10975146 (Y.B.). The author is also grateful to the CAS for grant No KJCX3-SYW-N2. B.D. would like to thank IHEP and LPSC for offering hospitality allowing him to achieve this work. He is also very grateful for support from valencian colleagues, which allowed him to attend the LC2010 conference.

\section{References}

[1] P.A.M. Dirac, Rev. Mod. Phys. 21 (1949) 392.

[2] B. Bakamjian, L.H. Thomas, Phys. Rev. 92 (1953) 1300.

[3] B. Keister, W. Polyzou, Adv. Nucl. Phys. 20 (1991) 225.

[4] S.N. Sokolov, A.N. Shatnii, Theor. Math. Phys. 37 (1978) 1029.

[5] F.M. Lev, Rivista del Nuovo Cimento 16 (1993) 1.

[6] B. Desplanques, Yu-bing Dong, [nucl-th/1006.0578].

[7] B. Desplanques, Yu-bing Dong, [nucl-th/0907.2835].

[8] B. Desplanques, Yu-bing Dong, Eur. Phys. J. A 37 (2008) 33.

[9] B. Desplanques, Eur. Phys. J. A 42, (2009) 219.

[10] B. Desplanques, L. Theuß1, Eur. Phys. J. A 21 (2004) 93.

[11] B. Bakamjian, Phys. Rev. 121 (1961) 1849.

[12] B. Desplanques, Nucl. Phys. A 748 (2005) 139.

[13] D. Melikhov, Eur. Phys. J. direct C4 (2002) 2 [hep-ph/0110087].

[14] A.F. Krutov, V.E. Troitsky, Phys. Rev. C 65 (2002) 045501.

[15] S. Simula, Phys. Rev. C 66, 035201 (2002).

[16] B. Bakker, H.-M. Choi, C.-R. Ji, Phys. Rev. D 63, 074014 (2001).

[17] J. P. B. C. de Melo et al., Nucl. Phys. A 707, 399 (2002).

[18] F. Cardarelli et al., Phys. Lett. B 332, 1 (1994).

[19] F. Cardarelli, E. Pace, G. Salmé, S. Simula, Phys. Lett. B 357, 267 (1995).

[20] J. P. B. C. de Melo et al., Nucl. Phys. A 782, 69 (2007).

[21] F. Gross, G. Ramalho, M.T. Pena, Phys. Rev. C 77, 015202 (2008). 